\title{
Proton lateral broadening distribution comparisons between GRNTRN, MCNPX, and laboratory beam measurements
}

\author{
Christopher J. Mertens ${ }^{\mathrm{a},}{ }^{,}$, Michael F. Moyers ${ }^{\mathrm{b}}$, Steven A. Walker ${ }^{\mathrm{c}}$, and John Tweed ${ }^{\mathrm{c}}$ \\ ${ }^{a}$ NASA Langley Research Center, Hampton, Virginia 23681-2199, United States \\ b Proton Therapy, Inc., Colton, California 92324, United States \\ c Old Dominion University, Norfolk, Virginia 23529, United States
}

\begin{abstract}
Recent developments in NASA's deterministic High charge (Z) and Energy TRaNsport (HZETRN) code have included lateral broadening of primary ion beams due to smallangle multiple Coulomb scattering, and coupling of the ion-nuclear scattering interactions with energy loss and straggling. This new version of HZETRN is based on Green function methods, called GRNTRN, and is suitable for modeling transport with both space environment and laboratory boundary conditions. Multiple scattering processes are a necessary extension to GRNTRN in order to accurately model ion beam experiments, to simulate the physical and biological-effective radiation dose, and to develop new methods and strategies for light ion radiation therapy. In this paper we compare GRNTRN simulations of proton lateral broadening distributions with beam measurements taken at Loma Linda University Proton Therapy Facility. The simulated and measured lateral broadening distributions are compared for a $250 \mathrm{MeV}$ proton beam on aluminum, polyethylene, polystyrene, bone substitute, iron, and lead target materials. The GRNTRN results are also compared to simulations from the Monte Carlo MCNPX code for the same projectile-target combinations described above.
\end{abstract}

\section{Introduction}

GReeN function TRaNsport (GRNTRN) is a new version of NASA's deterministic High charge $(Z)$ and Energy TRaNsport (HZETRN) code that is based on non-perturbative Green function techniques for the transport of charged particles through target media (Tweed et al., 2004, 2005). The computational transport procedures in HZETRN were developed for space environment boundary conditions consisting of omni-directional particle flux with continuous energy spectra (Wilson et al., 1991). Ground-based laboratory beam experiments, on the other hand, are described by the transport of directed-beams of ions with quasi-monochromic energy. The advantage of the Green function approach, and the motivation for GRNTRN development, is the ease and flexibility by which both ground-based laboratory and space environment boundary conditions can be incorporated into a single theoretical formulation. Moreover, the Green function method enables detailed simulations of the response of particle spectrometer devices, which are used to diagnose laboratory beam experiments (Walker et al., 2005).

Because ion beams in laboratory experiments are quasi mono-directional, incorporating off-axis dispersion of the primary ion beam is an important feature that needs to be 
included in ion beam transport codes. The beam broadening mechanism included in GRNTRN is attributed to small-angle multiple Coulomb scattering of the incident ion beam by the target media nuclei (Mertens et al., 2007). While the effects of multiple scattering are negligible in the omni-directional space radiation environment, multiple scattering effects must be included in directed-beam applications and ground-based laboratory transport code simulations.

Mertens et al. (2007) presented the theoretical formulation and computation procedures for incorporating small-angle multiple Coulomb scattering into the GRNTRN code. The GRNTRN multiple scattering interactions and transport include a self-consistent coupling of ion-nucleus scattering with ionization energy loss and straggling. Initial benchmark studies included comparisons of beam broadening characteristics with Monte Carlo simulations and laboratory experiments reported by Noshad and Givechi (2005) for a 60 $\mathrm{MeV}$ proton beam on muscle tissue.

In this paper we expand upon our initial benchmark studies and compare proton lateral broadening distributions with beam experiments conducted by Moyers (2005) at the Loma Linda University Proton Treatment Facility (LLUPTF). In the LLUPTF experiments, a $250 \mathrm{MeV}$ proton beam was incident upon phantom targets representative of low- $\left(\leq 1 \mathrm{~g} / \mathrm{cm}^{3}\right)$, medium- $\left(1-5 \mathrm{~g} / \mathrm{cm}^{3}\right)$, and high-density $\left(>5 \mathrm{~g} / \mathrm{cm}^{3}\right)$ materials. The low-density materials are air, high-density polyethylene (HDPE), and clear cross-linked polystyrene (CLPS). The medium-density materials are RMI bone and aluminum (Al). The high-density materials are iron $(\mathrm{Fe})$ and lead $(\mathrm{Pb})$. We compare GRNTRN/LLUPTF lateral broadening distributions for all proton-target materials listed above. Moreover, we compare the Monte Carlo MCNPX code simulations of the lateral beam broadened fullwidth at half-max (FWHM) reported by Moyers (2005) with corresponding GRNTRN simulations for the same proton-target combinations described above.

\section{GRNTRN Multiple Scattering Formulation}

In this section we outline the main features of the transport methodology implemented in GRNTRN for computing the lateral broadening distribution of the primary ion beam due to small-angle multiple Coulomb ion-nuclear scattering. The details of the theoretical formulation and computational procedures were presented in an earlier report (Mertens et al., 2007).

At LLUPT, the lateral broadening distributions were measured with an extended dose range radiographic film. To compare with these measurements, we computed the off-axis dose distributions by factoring it into an on-axis dose distribution modulated by an offaxis beam broadening function (Hogstrom et al., 1981), such that

$$
d(x, y, z)=f(x, y, z) d(z),
$$

where $d(z)$ is the on-axis dose distribution and $f(x, y, z)$ is the off-axis beam broadening function. The on-axis dose distribution is given by the expression 


$$
d(z)=K \int_{0}^{\infty} S(E) \Phi(z, E) d E
$$

where $\mathrm{S}(\mathrm{E})$ is the stopping power, $\Phi(z, E)$ is the on-axis spectral flux distribution computed from a second-order energy moment expansion of the Boltzmann transport equation (Wilson et al., 2000, 2002), and $K$ is a unit conversion factor. Assuming cylindrical symmetry, the off-axis broadening function is given by

$$
f(x, y, z)=\frac{1}{\pi\left\langle r^{2}(z)\right\rangle} \exp \left(-\frac{\left(x^{2}+y^{2}\right)}{\left\langle r^{2}(z)\right\rangle}\right) .
$$

Moreover, the transport integral for the radial broadening statistical moment is

$$
\left\langle r^{2}(z)\right\rangle=\left\langle r^{2}(0)\right\rangle+\int_{0}^{z}\left(z-z^{\prime}\right)^{2} \frac{d\left\langle\theta^{2}\left(z^{\prime}\right)\right\rangle}{d z^{\prime}} d z^{\prime}
$$

In the above equation, $\left\langle r^{2}(0)\right\rangle$ is the initial radial broadening of the incident ion beam, which assumes there are no initial angular broadening and radial-angular correlation in the incident ion beam - i.e., all incident ions are propagating parallel to one another. The scattering power in (4) is given by

$$
\begin{aligned}
\frac{d\left\langle\theta^{2}(z)\right\rangle}{d z}= & 4 \pi Z_{P}^{2} \frac{\left(r_{e} \mu_{e}\right)^{2}}{(\mathrm{v}(z) p(z))^{2}} \sum_{m} \rho_{A}(m) Z_{T}(m)\left(Z_{T}(m)+1\right) \\
& \times\left\{\ln \left[\theta_{r}^{2}(m)+1\right]-1+\left[\theta_{r}^{2}(m)+1\right]^{-1}\right\},
\end{aligned}
$$

where

$$
\theta_{r} \equiv \frac{\theta_{u}}{\theta_{l}}=\left[\left.181 Z_{T}^{-1 / 3}\left(\frac{Z_{T}}{A_{T}}\right)^{1 / 6}\right|^{2} .\right.
$$

In equations (5)-(6), the variables are defined as follows: $A_{T}$ is the target atomic mass number, $Z_{T}$ and $Z_{P}$ are the target and projectile atomic charge numbers, respectively, $r_{e}$ is the classical electron radius, $\mu_{e}$ is the electron rest mass, $\mathrm{v}$ and $p$ are the projectile velocity and momentum, respectively, $\rho_{A}$ is the number of atoms per gram, $\theta_{u}$ and $\theta_{l}$ are the upper and lower limits of the scattering angle consistent with small-angle Coulomb scattering, and the summation is over $\mathrm{m}$, the elemental constituents of the target material. 
Ionization energy loss is implicitly coupled to the multiple scattering transport through the depth dependence of the projectile velocity and momentum in (5). Assuming the continuous slowing down approximation, the mean energy of the ion beam after propagating a longitudinal distance $z$ into the target material is given by the usual rangeenergy relations (Wilson et al., 1991). The velocity and momentum of the projectile at each propagation depth- $z$ in (5) can be evaluated using the mean energy and the relativistic kinematic relations.

In order to achieve a self-consistent coupling between multiple scattering transport and energy loss, the influence of multiple scattering on the mean energy must also be included, in addition to the influence of energy loss on multiple scattering implicit in (5). Multiple scattering influences ionization energy loss due to the fact that the actual path length of the projectile beam particles is greater than the physical depth- $z$ in the material. An effective 1-D path length correction, due to multiple scattering, is introduced into the range-energy relation such that the mean energy is given by

$$
\bar{E}(z)=R^{-1}\left[R\left(E_{0}\right)-(z+\langle\Delta R(z)\rangle)\right]
$$

where the unit of energy is $\mathrm{MeV} /$ nucleon. The above equation describes, in operator form, the inverse of the range-energy relation to obtain the mean energy. The mean range of the incident beam at the initial energy $E_{0}$ at zero depth is denoted $R\left(E_{0}\right)$, and the effective 1-D multiple scattering path length correction is denoted $\langle\Delta R(z)\rangle$. Thus, the term in brackets in (7) is the residual range, and the (residual) mean energy is found by interpolating with respect to the residual range within a pre-computed database of range versus energy.

The path length correction is expressed as an integral of the lateral projection of meansquared scattering angle over depth (Bichsel and Uehling, 1960), which, assuming cylindrical symmetry, is given by

$$
\langle\Delta R(z)\rangle=\int_{0}^{z}\left\langle\theta_{x}^{2}\left(z^{\prime}\right)\right\rangle d z^{\prime}=\int_{0}^{z}\left\langle\theta_{y}^{2}\left(z^{\prime}\right)\right\rangle d z^{\prime}
$$

Mertens et al. (2007) showed that the lateral projection of mean-squared scattering angle at intermediate depths- $z^{\prime}$ depends on the two boundary conditions at zero depth and at depth-z, which is given in terms of a doubly-conditional probability distribution

$$
\left\langle\theta_{y}^{2}\left(z^{\prime}\right)\right\rangle=\int_{-\infty}^{+\infty} P\left(\theta_{y}^{\prime} ; z^{\prime} \mid 0, y_{0}, \theta_{0} ; z, y_{1}, \theta_{1}\right) \theta_{y}^{2} d \theta^{\prime}
$$

The probability function above is defined such that at depth- $z^{\prime}$ the ion beam has a probability of having an angular displacement $\theta_{y}^{\prime}$ with respect to the y-axis, given that the beam had lateral and angular displacements $y_{0}$ and $\theta_{0}$, respectively, at zero depth, and emerges at depth-z with lateral and angular displacements $y_{1}$ and $\theta_{1}$, respectively. For the 
purpose of calculating an effective 1-D path length correction, we can consider the initial beam to have zero lateral and angular displacements and integrate over all possible emerging lateral and angular displacements. Thus, the final expression for the path length correction is (Mertens et al., 2007)

$$
\langle\Delta R(z)\rangle=\frac{1}{2} \int_{0}^{z} d z^{\prime} \int_{0}^{z^{\prime}} \frac{d\left\langle\theta^{2}\left(z^{\prime \prime}\right)\right\rangle}{d z^{\prime \prime}} d z^{\prime \prime}
$$

Equations (7) and (10), combined with the relativistic kinematic relations, comprise a self-consistent depth-dependent specification of the scattering power in (5), which enables a complete description of the broadened beam characteristics such as lateral beam distribution function in (3) and the mean-square radial broadening statistical moment in (4).

\section{Results and Discussion}

In this section we present comparisons of lateral broadening distributions calculated from GRNTRN with measurements taken at LLUPTF for a $250 \mathrm{MeV}$ proton beam incident on various target material slabs. Moreover, we compare GRNTRN and MCNPX simulations of the lateral distribution's FWHM for the same target materials as the LLUPTF/GRNTRN comparisons.

An overview of the LLUPTF experimental set-up is as follows (Moyers, 2005). The protons were accelerated to $250 \mathrm{MeV}$ by a synchrotron and transported in a vacuum pipe to a research cave. After entering the cave, the beam exited the vacuum pipe through a titanium window. The beam passed through several centimeters of air before entering a transmission ionization chamber (TIC), which consisted of five elements. The upstream TIC element was a single collecting layer that intercepted the entire beam to provide a normalizing signal. The next downstream TIC element was a layer segmented into four quadrants to provide beam-centering information. The target slab materials for the scattering measurements were placed downstream of the TIC. Table 1 lists the target materials, their densities, their nominal slab thicknesses, and the number of slabs for each target material used in the experiments.

The lateral distributions were measured using extended dose range radiographic films placed perpendicular to the incident beam at various distances along the beamline. Before each target material experiment, films were exposed immediately downstream of the TIC and at approximately $88 \mathrm{~cm}$ downstream of the TIC in order to determine the spot size, shape, and divergence of the incident beam. These results were used to define the lateral width of the beam source in the transport calculations. Also, the measured spacing and elemental composition of all elements along the beamline (e.g., the titanium vacuum window, the five TIC elements, target materials, etc.) were included in the transport calculations. Although the measured beam shape exiting the vacuum pipe was slightly elliptical in shape, with the long axis of the ellipse usually oriented at 45 degrees with respect to the horizontal plane, a circular beam shape was assumed in both the GRNTRN and MCNPX simulations. 
Figure 1 shows the LLUPTF experimental set-up for the lateral broadening measurements in air, and Figure 2 shows the corresponding measurement and GRNTRN simulation results. In the set-up shown in Figure 1, the lateral distribution in relative dose was measured immediately downstream of the TIC, at $-5.0 \mathrm{~cm}$ with respect to the beamline reference point, and at $82.7 \mathrm{~cm}$ downstream of the reference point. The measured FWHM at $-5.0 \mathrm{~cm}$ and $82.7 \mathrm{~cm}$ was $0.29 \mathrm{~cm}$ and $0.62 \mathrm{~cm}$, respectively (see Table 2). The lateral broadening in relative dose at $-5.0 \mathrm{~cm}$ is the result of the finite width of the initial beam incident at the vacuum window and subsequent scattering due to the small amount of material in the vacuum window, the TIC, and air between the vacuum window and the TIC. The distance in air between the film measurement at $-5.0 \mathrm{~cm}$ and at $82.7 \mathrm{~cm}$ is a drift space which amplifies the scattering effects, as is evident in Figure 2 by the increased lateral broadening in relative dose at $82.7 \mathrm{~cm}$ with respect to the broadening at $-5.0 \mathrm{~cm}$. By comparing ratios of GRNTRN and LLUPTF FWHM, GRNTRN overestimates the scattering with respect to the beam measurements by $26 \%$ at $82.7 \mathrm{~cm}$, as shown in Table 2.

A sandwich experimental configuration is used for the low- and medium-density target materials, as shown in Figure 3 for the CLPS set-up. The target slab materials are placed at three locations downstream of the beamline reference point, and the target slabs are separated by foam slabs. The foam slabs are low-density materials that provide a drift space to magnify the scattering effects. The experiments for the HDPE, bone, and Al target slabs use the same set-up as shown in Figure 3 for the CLPS slabs.

Figures 4-7 show the comparisons between the lateral broadening in relative dose between the LLUPTF film measurements and GRNTRN simulations for CLPS, HDPE, bone, and Al, respectively. Similar to the results for transport through air shown in Figure 2, GRNTRN appears to overestimate the multiple scattering effects for low- and medium density target materials. For easy comparison, Table 2 provides ratios of the FWHMs determined by the different methods. The largest GRNTRN/LLUPTF FWHM ratios are observed for the low-density materials. Thus, the largest FWHM overestimation between GRNTRN and LLUPTF is $38 \%$ at $27.6 \mathrm{~cm}$ for the CLPS set-up. The ratios in simulated versus measured FWHM are within $14 \%$ for the medium density materials (bone and $\mathrm{Al}$ ).

The high-density target material experiments use an effective single slab set-up, which is shown in Figure 8. The thin high-density layers (see Table 1) are stacked into a single slab and placed in the apparatus attached to the TIC. Downstream of the TIC are foam slabs used to provide the drift space. Two measurements are made for the high-density target set-ups: one measurement at $-5.0 \mathrm{~cm}$ and one at about $69 \mathrm{~cm}$. The LLUPTF and GRNTRN lateral distributions in relative dose for $\mathrm{Fe}$ and $\mathrm{Pb}$ are shown in Figures 9 and 10, respectively. From Table 2, GRNTRN simulations of FWHM overestimate the LLUPTF measurements by no more than $16 \%$ for the high-density target materials.

The results in Figures 2, 4-7, 9-10 and Table 2 show that GRNTRN systematically overestimates the scattering effects relative to the LLUPTF measurements. In comparing MCNPX lateral broadening simulations with LLUPTF measurements, Moyers (2005) considered the possibility that the real proton beam is slightly converging. Simulations 
indicated that if the beam were in vacuum the lateral FWHM would decrease at a rate of $0.20-0.28 \mathrm{~cm}$ per meter. At $88 \mathrm{~cm}$ downstream of the TIC, the lateral FWHM would decrease by $0.18-0.25 \mathrm{~cm}$. Comparing the LLUPTF and GRNTRN FWHM differences in Table 2 (columns 8-9), we see that the estimated convergence of the real beam may explain the measurement versus simulated differences in air, but does not explain the differences in the other target materials, especially for the high-density target materials. Thus, the measured versus simulated differences in lateral FWHM is larger than can be accounted for by the estimated convergence of the real proton beam.

Moyers (2005) compared results of transport simulation using the Monte Carlo code MCNPX v.2.5 with LLUPTF lateral beam distributions and FWHM measurements. MCNPX uses the Rossi (1952) formalism for scattering. Later, Moyers et al. (2005) repeated the MCNPX simulations but changed the numerical value of the scattering parameter $\alpha$ (Prael, 2002) from 1/137 to 1/50. We included both MCNPX results in Table 2 for comparisons with GRNTRN and measurements. Both the standard MCNPX and GRNTRN transport algorithms systematically overestimate the lateral FWHMs compared to measurement data and have comparable discrepancies. The largest discrepancies are for the low-density target materials (CLPS and HDPE). The largest discrepancy between MCNPX and LLUPTF measurements is $34 \%$ for CLPS. The modified MCNPX gave much better agreement for these experimental conditions. The comparison for large thicknesses showed the modified MCNPX slightly overestimated the FWHM for loawand high-atomic number and density materials and slightly underestimated the FWHM for intermediate atomic number and density materials. Although this parameter change was not based upon any physics, these simulations do serve to illustrate how sensitive the calculations are to a single parameter.

We consider a number of possible explanations for the apparent overestimation of the scattering effects on beam transport for the GRNTRN and standard MCNPX simulations. The assumption of cylindrical symmetry in the model simulations does not appear to be a viable explanation. Although the real beam was determined to be elliptical, the eccentricity is small since the measured distributions along the two mutually perpendicular lateral directions are roughly the same for each proton-target combination (see Figures 1-10). Single scattering events would broaden the wings of the lateral distributions. The measured lateral distributions are fairly well represented by Gaussians, consistent with our modeling assumption of small-angle multiple scattering events. The simulations agree well with measurements immediately downstream of the TIC at -5.0 $\mathrm{cm}$, indicating that the transport codes adequately model the beamline components prior to the target materials. Since the GRNTRN and MCNPX results are comparable, and yet based on vastly different transport methodologies, it appears that the transport physics is not a likely source for the measurement and model discrepancies.

A plausible explanation for the discrepancy between the measured lateral broadening characteristics and the corresponding simulations from the GRNTRN and MCNPX codes is inaccuracies in the basic ion-nucleus scattering cross section. This explanation is supported by the greatly improved agreement between measurements and simulations after the change of a single parameter in MCNPX. Both GRNTRN and MCNPX transport 
models use a screened Coulomb potential to represent the interaction between the ion beam and target nucleus, where the Thomas-Fermi statistical model is used to define the screening radius (Mertens et al., 2007). The Thomas-Fermi model becomes progressively less accurate for low- $Z$ target nuclei. This is consistent with transport model comparisons with the LLUPTF measurements in Table 2. The largest measurement versus model discrepancies occur for the low-density target materials with sufficient concentrations of low-Z atoms, while the best agreement occurs for the medium- to high-density materials composed of mid- to high- $Z$ atoms. Repeated comparisons with explicit improvements in the screened Coulomb potential for low- $\mathrm{Z}$ atoms are required to be definitive about this conclusion.

\section{Summary}

The new ion beam small-angle multiple Coulomb scattering formulation implemented in GRNTRN has been tested by comparisons with measurements of lateral beam broadening characteristics taken at the Loma Linda University Proton Treatment Facility for a 250 $\mathrm{MeV}$ proton beam on low-, medium-, and high-density target materials. The GRNTRN results versus measurement data are in fairly good agreement. GRNTRN simulations of the lateral beam distribution's FWHM is within $15 \%$ of the measured results for mediumand high-density target materials. The largest discrepancies occur for the low-density and low-Z target materials with a maximum GRNTRN overestimation of 38\% in FWHM for the CLPS target. GRNTRN results are comparable to predictions from standard Monte Carlo MCNPX simulations. Both transport algorithms systematically overestimate the scattering effects. The largest discrepancies between measurement data and the transport algorithms are for the low-density and low-Z target materials, suggesting that the source of the discrepancies may be inaccuracies in the parameterizations of the screened Coulomb potential utilized in the ion-nucleus scattering cross section. Improved scattering cross sections and continued comparisons between transport algorithms and ion beam measurements are required to definitively assess the accuracy of the transport code's multiple scattering formulation, which is the topic of a future report. Nevertheless, the current version of GRNTRN appears to be well-suited for analysis of ion beam experiments, radiobiology studies, and the development of light-ion therapy strategies. 


\section{References}

Bichsel, H., and E. D. Uehling, Multiple scattering correction for proton ranges and the evaluation of the L-shell correction and I value for Aluminum, Phys. Rev., 119(5), $1670-1680,1960$.

Hogstrom, K. R., M. D. Mills, and R. Almond, Electron beam dose calculations, Phys. Med. Biol., 26(3), 445-459, 1981.

Mertens, C. J., J. W. Wilson, S. A. Walker, and J. Tweed, Coupling of multiple Coulomb scattering with energy loss and straggling in HZETRN, Adv. Space Res., 40, $1357-$ 1367, 2007.

Moyers, M. F., Comparison of measured and MCNPX calculated lateral scattering distributions for $250 \mathrm{MeV}$ protons, Space Nuclear Conference 2005: Embedded Topical Meeting Proceedings (Illinois: American Nuclear Society, Inc., 2005) p. 635-644. ISBN:0-89448-696-9, 2005.

Moyers, M. F., M. F. James, G. W. McKinney, Prediction of lateral proton scattering by MCNPX, Particle Therapy Co-operative Group meeting, Munich, Germany, December 9-14, 2005. (unpublished).

Noshad, H., and N. Givechi, Proton therapy using the Monte Carlo method, Radiat. Meas., 39, 521-524, 2005.

Prael, R. E., Multiple coulomb scattering methods in LAHET3 and MCNPX, Los Alamos Research Note (U) 01-59 LA-UR-02-0425 Los Alamos National Laboratory, New Mexico, 2002.

Rossi, B. High-energy Particles (New Jersey, Prentice-Hall, Inc., 1952)

Tweed, J., J. W. Wilson, and R. K. Tripathi, An improved Green function for ion beam transport, Adv. Space. Res., 34, 1311-1318, 2004.

Tweed, J., S. A. Walker, J. W. Wilson, F. A. Cucinotta, R. K. Tripathi, S. Blattnig, and C. J. Mertens, Computation methods for the HZETRN code, Adv. Space Res., 25, 194$201,2005$.

Walker, S. A., J. Tweed, J. W. Wilson, F. A. Cucinotta, R. K. Tripathi, S. Blattnig, C. Zeitlin, L. Heilbronn, and J. Miller, Validation of the HZETRN code for laboratory exposures with $1 \mathrm{~A} \mathrm{GeV}$ iron ions in several targets, Adv. Space Res., 35, 202-207, 2005.

Wilson, J. W., J. Tweed, H. Tai, and R. K. Tripathi, A simple model for straggling evaluation, Nucl. Instrum. Methods B, 194, 389-392, 2002.

Wilson, J. W., and H. Tai, Range and energy straggling in ion beam transport, NASA/TP-2000-209864, 2000.

Wilson, J. W., L. W. Townsend, W. Schimmerling, G. S. Khandelwal, F. Khan, J. E. Nealy, F. A. Cucinottam and L. C. Simonsen, J. L. Shinn, and J. W. Norbury, Transport Methods and Interactions for Space Radiations, Tech. Rep. NASA RP1257, 1991. 
Table 1: Target Materials for Beam Experiments (Moyers, 2005)

\begin{tabular}{|l|l|l|l|l|}
\hline Material & Abbreviation & $\begin{array}{l}\text { Density } \\
\left(\mathbf{g} / \mathbf{c m}^{\mathbf{3}}\right)\end{array}$ & $\begin{array}{l}\text { Nominal Slab } \\
\text { Thickness }(\mathbf{c m})\end{array}$ & $\begin{array}{l}\text { Number of } \\
\text { Slabs }\end{array}$ \\
\hline Air & Air & 0.0012 & N/A & N/A \\
\hline $\begin{array}{l}\text { High density } \\
\text { polystyrene } \\
\text { foam }\end{array}$ & Foam & 0.031 & 7.6 & 9 \\
\hline $\begin{array}{l}\text { High density } \\
\text { polyethylene }\end{array}$ & HDPE & 0.963 & 5 & 3 \\
\hline $\begin{array}{l}\text { Clear cross- } \\
\text { linked } \\
\text { polystyrene }\end{array}$ & CLPS & 1.041 & 5 & 3 \\
\hline RMI bone & Bone & & 3 & 3 \\
\hline Aluminum & Al & 1.814 & 2 & 3 \\
\hline Iron & Fe & 2.683 & 2 & 6 \\
\hline Lead & Pb & 7.720 & 0.15 & 2 \\
\hline
\end{tabular}


Table 2: Comparison of GRNTRN (GN), LLUPTF Beam Measurements (BM), and MCNPX (MC) Lateral Broadening Width (FWHM) of 250 MeV Proton Beam on the target material slabs below.

\begin{tabular}{|c|c|c|c|c|c|c|c|c|c|c|c|}
\hline \multirow[t]{2}{*}{$\begin{array}{l}\text { Target Material / } \\
\text { Number of Slabs }\end{array}$} & \multirow{2}{*}{$\begin{array}{c}\text { Distance from } \\
\text { Reference } \\
\text { [cm] }\end{array}$} & \multicolumn{10}{|c|}{$\begin{array}{c}\text { FWHM } \\
\text { [cm] }\end{array}$} \\
\hline & & $\mathbf{B M}$ & GN & MC & $\mathbf{M C}_{\alpha}$ & GN/BM & МС/BM & $\mathrm{MC}_{\alpha} / \mathrm{BM}$ & GN-BM & MC-BM & $\mathrm{MC}_{\alpha}-\mathrm{BM}$ \\
\hline \multirow[t]{2}{*}{ Air } & -5.0 & 0.29 & 0.29 & 0.29 & 0.29 & 1.00 & 1.00 & 1.00 & 0.00 & 0.00 & 0.00 \\
\hline & +82.7 & 0.62 & 0.78 & 0.76 & 0.70 & 1.26 & 1.23 & 1.13 & 0.16 & 0.14 & 0.08 \\
\hline HDPE & -5.0 & 0.28 & 0.25 & 0.27 & 0.27 & 0.89 & 0.96 & 0.96 & -0.03 & -0.01 & -0.01 \\
\hline 1 & +27.8 & 0.61 & 0.80 & 0.74 & 0.66 & 1.31 & 1.21 & 1.08 & 0.19 & 0.13 & 0.05 \\
\hline 2 & +55.6 & 1.55 & 1.85 & 1.84 & 1.51 & 1.19 & 1.19 & 0.97 & 0.30 & 0.29 & -0.04 \\
\hline 3 & +83.4 & 2.58 & 3.24 & 2.80 & 2.57 & 1.26 & 1.09 & 1.00 & 0.66 & 0.22 & -0.01 \\
\hline CLPS & -5.0 & 0.23 & 0.23 & 0.25 & 0.23 & 1.00 & 1.09 & 1.00 & 0.00 & 0.02 & 0.00 \\
\hline 1 & +27.8 & 0.58 & 0.80 & 0.78 & 0.70 & 1.38 & 1.34 & 1.21 & 0.22 & 0.20 & 0.12 \\
\hline 2 & +55.6 & 1.47 & 1.86 & 1.74 & 1.56 & 1.26 & 1.18 & 1.06 & 0.39 & 0.27 & 0.09 \\
\hline 3 & +83.4 & 2.56 & 3.27 & 3.05 & 2.70 & 1.28 & 1.19 & 1.05 & 0.71 & 0.49 & 0.14 \\
\hline Bone & -5.0 & 0.28 & 0.25 & 0.27 & 0.27 & 0.89 & 0.96 & 0.96 & -0.03 & -0.01 & -0.01 \\
\hline 1 & +25.8 & 0.85 & 0.92 & 0.98 & 0.88 & 1.08 & 1.15 & 1.04 & 0.07 & 0.13 & 0.03 \\
\hline 2 & +51.6 & 1.85 & 2.09 & 2.09 & 1.82 & 1.13 & 1.13 & 0.98 & 0.24 & 0.24 & -0.03 \\
\hline 3 & +77.4 & 3.23 & 3.64 & 3.60 & 3.15 & 1.13 & 1.11 & 0.98 & 0.41 & 0.37 & -0.08 \\
\hline $\mathrm{Al}$ & -5.0 & 0.29 & 0.31 & 0.27 & 0.27 & 1.07 & 0.93 & 0.93 & 0.02 & -0.02 & -0.02 \\
\hline 1 & +25.0 & 0.85 & 0.97 & 0.91 & 0.90 & 1.14 & 1.07 & 1.06 & 0.12 & 0.06 & 0.05 \\
\hline 2 & +50.1 & 2.03 & 2.17 & 2.19 & 1.92 & 1.07 & 1.08 & 0.95 & 0.14 & 0.16 & -0.11 \\
\hline 3 & +75.1 & 3.50 & 3.73 & 3.77 & 3.31 & 1.06 & 1.08 & 0.95 & 0.23 & 0.27 & -0.19 \\
\hline $\mathrm{Fe}$ & -5.0 & 0.33 & 0.34 & 0.36 & 0.38 & 1.03 & 1.09 & 1.15 & 0.01 & 0.03 & 0.05 \\
\hline 1 & +69.6 & 3.74 & 4.33 & 4.39 & 3.83 & 1.16 & 1.17 & 1.02 & 0.59 & 0.65 & 0.09 \\
\hline $\mathrm{Pb}$ & -5.0 & 0.33 & 0.34 & 0.36 & 0.38 & 1.03 & 1.09 & 1.15 & 0.01 & 0.03 & 0.05 \\
\hline 1 & +69.3 & 3.75 & 4.30 & 4.55 & 3.88 & 1.15 & 1.21 & 1.03 & 0.55 & 0.80 & 0.13 \\
\hline
\end{tabular}




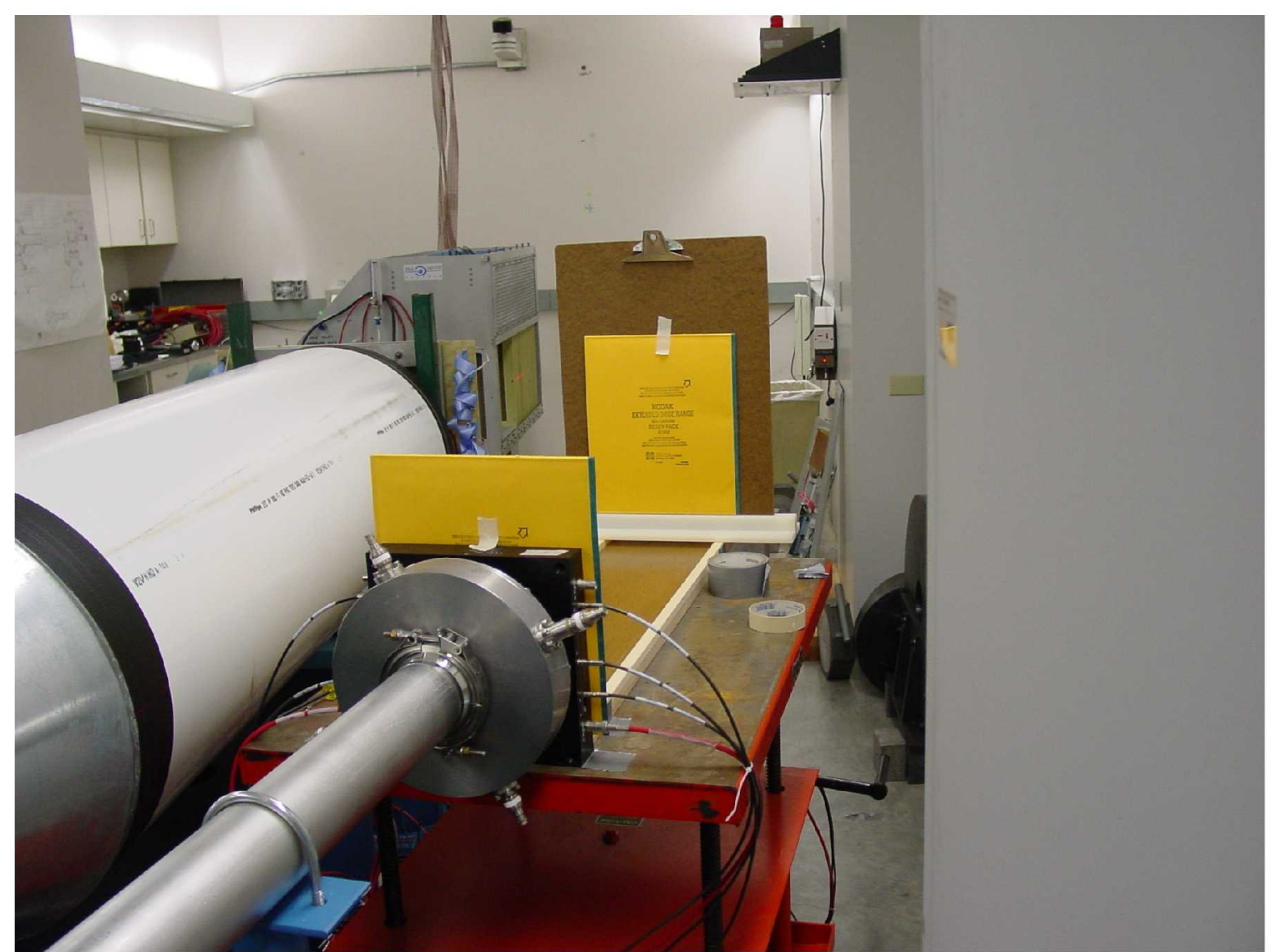

Figure 1: LLUPTF experimental set-up for proton broadening measurements in air. The radiographic film next to the TIC is at $-5.0 \mathrm{~cm}$ with respect to the beamline reference point. The film next to the clipboard is at $82.7 \mathrm{~cm}$ with respect to the reference point. 


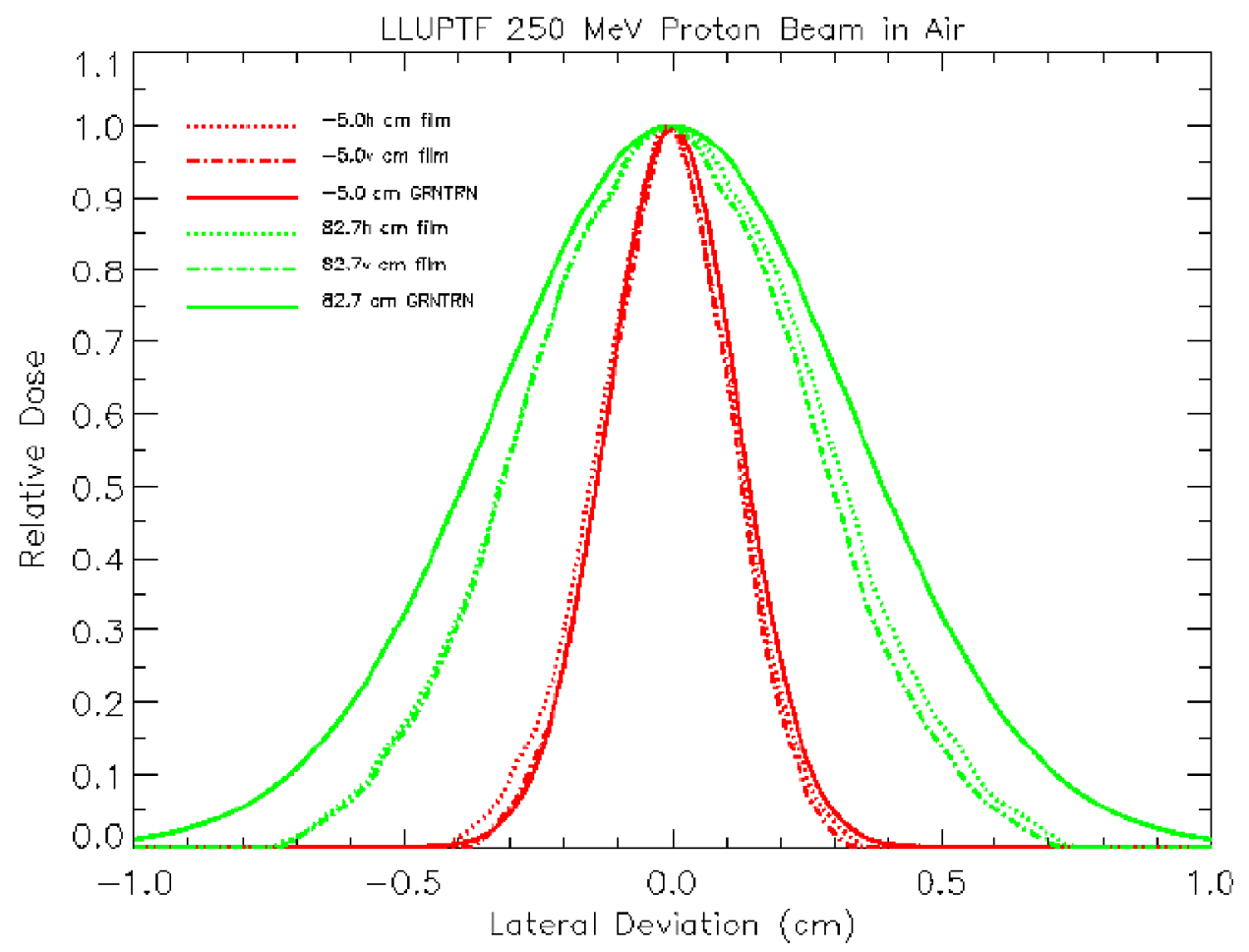

Figure 2: Lateral proton broadening in air. The red lines are results $5.0 \mathrm{~cm}$ upstream of the beamline reference point, which places the results immediately on the downstream side of the TIC. The green lines are results at $82.7 \mathrm{~cm}$ downstream of the reference point. See Figure 1 for picture of experimental set-up. The solid lines are GRNTRN simulations of the relative dose at lateral distances with respect to the beamline direction. The dotted and dashed lines are relative dose measured with the radiographic film in the two perpendicular (lateral) directions with respect to the beamline direction. 


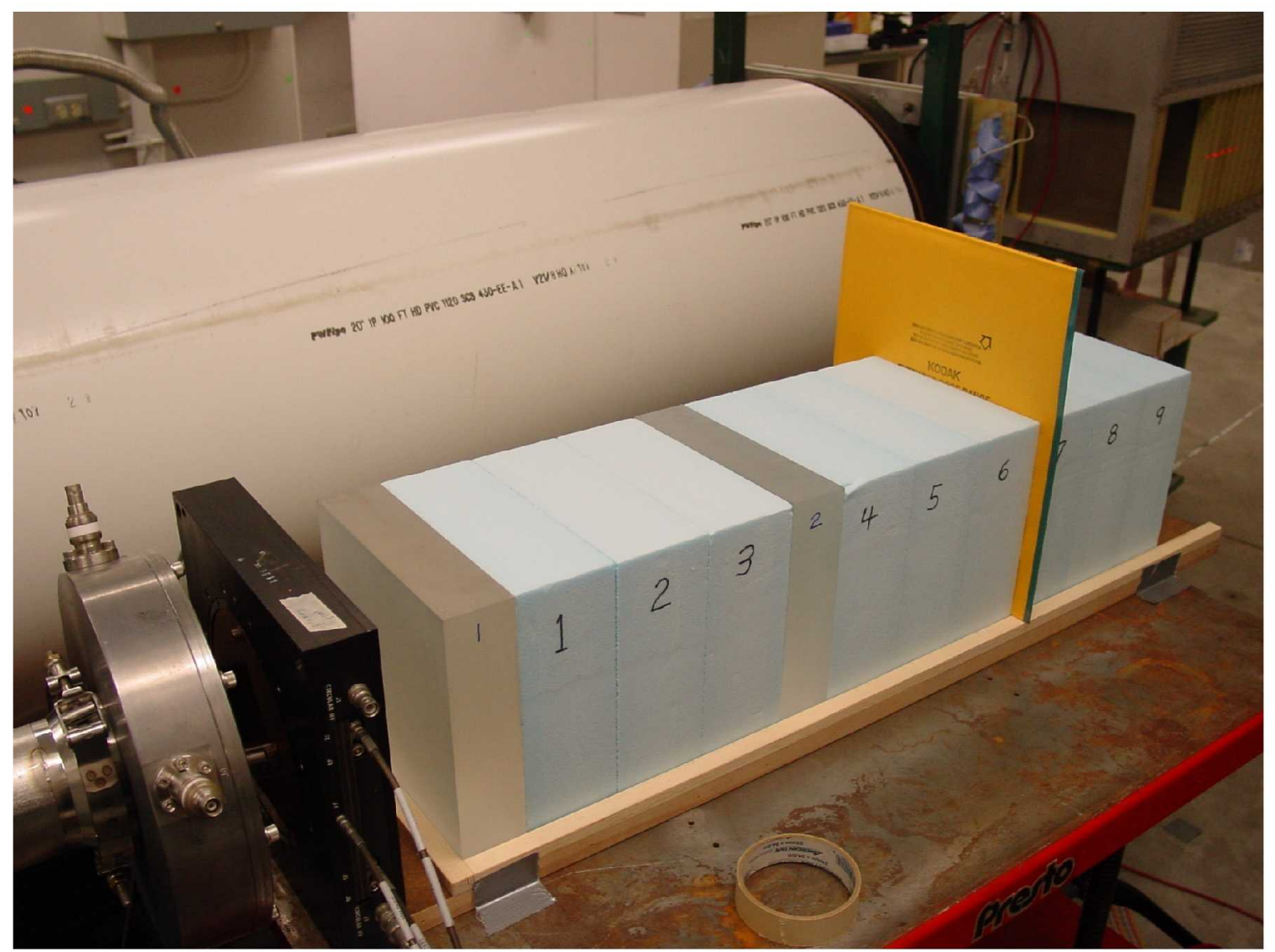

Figure 3: LLUPTF experimental set-up for proton broadening measurements in a sandwich configuration for CLPS slabs. The CLPS slabs are translucent and are marked as 1 and 2 in this picture. The CLPS slabs are separated by opaque foam slabs, which are marked 1-3, 4-6, and 7-9. In this picture the third CLPS slab is replaced by the radiographic film, which corresponds to the measurement at $55.6 \mathrm{~cm}$ in Figure 4 . 


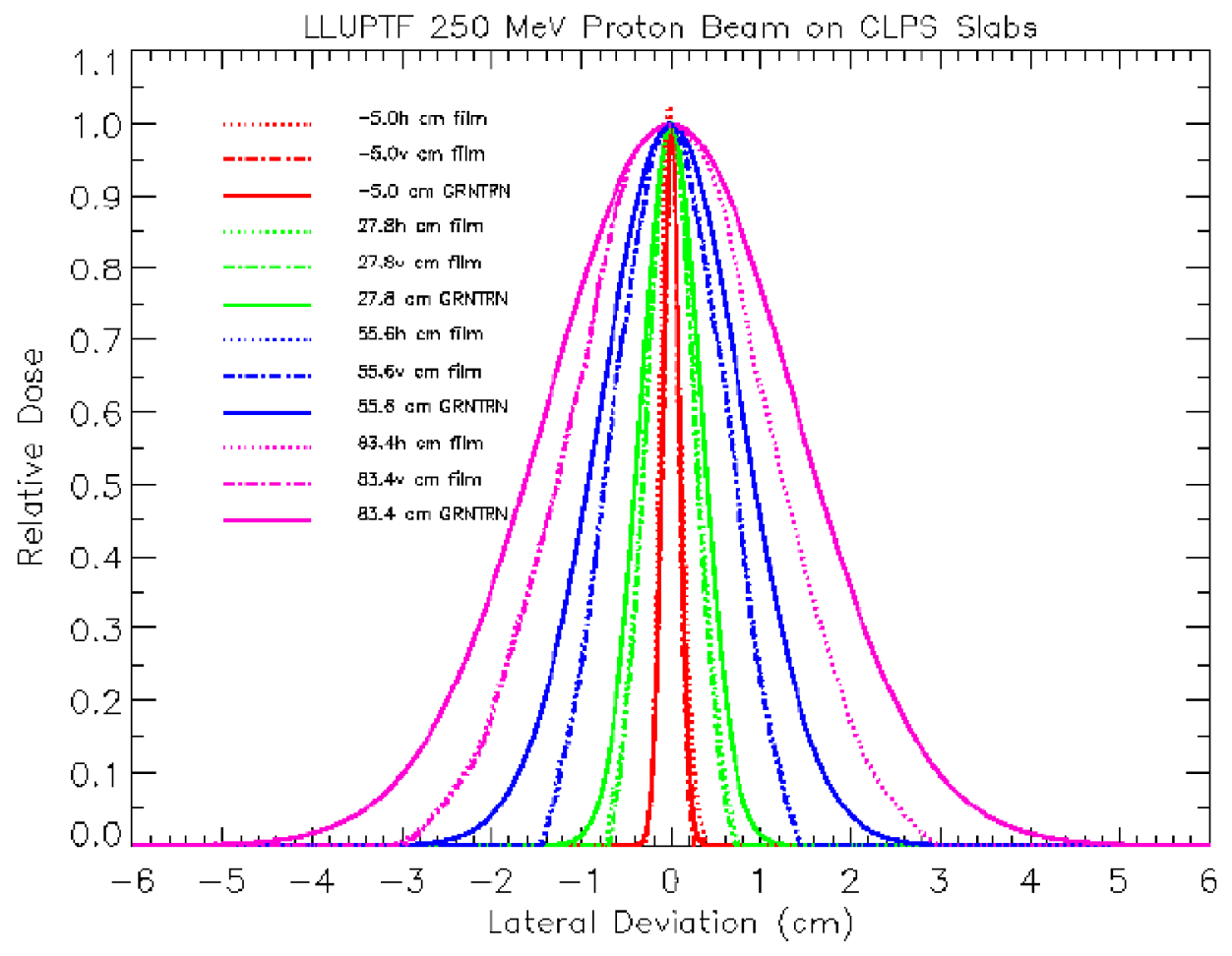

Figure 4: Lateral proton broadening in CLPS sandwich configuration (see Figure 3). The colored lines represent results at various distances along the beamline (see Figures 1-2 for beamline distance definitions). The solid lines are GRNTRN simulations of the relative dose at lateral distances with respect to the beamline direction. The dotted and dashed lines are relative dose measured with the radiographic film in the two perpendicular (lateral) directions with respect to the beamline direction. 


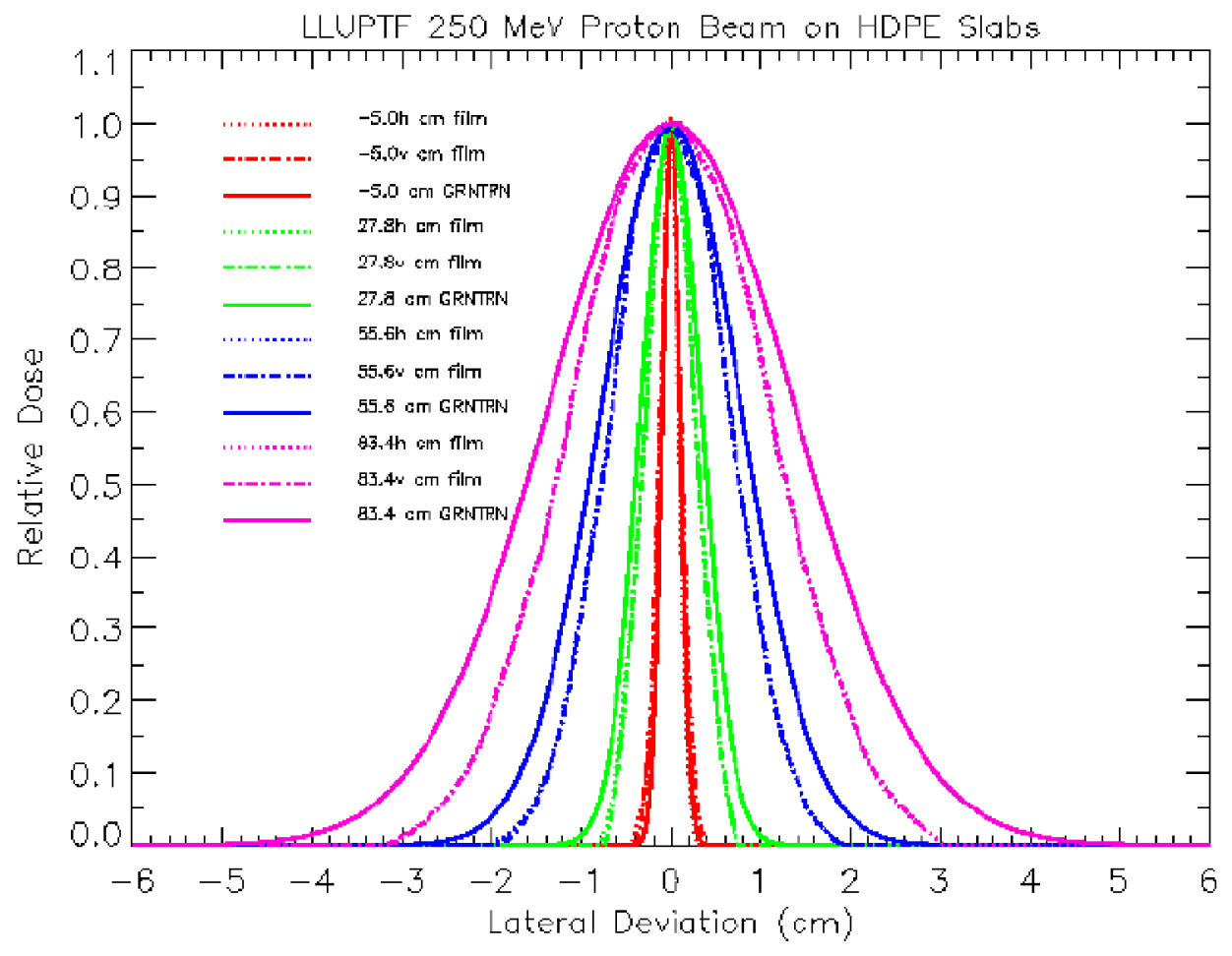

Figure 5: Lateral proton broadening in HDPE sandwich configuration (see Figure 3). The colored lines represent results at various distances along the beamline (see Figures 1-2 for beamline distance definitions). The solid lines are GRNTRN simulations of the relative dose at lateral distances with respect to the beamline direction. The dotted and dashed lines are relative dose measured with the radiographic film in the two perpendicular (lateral) directions with respect to the beamline direction. 


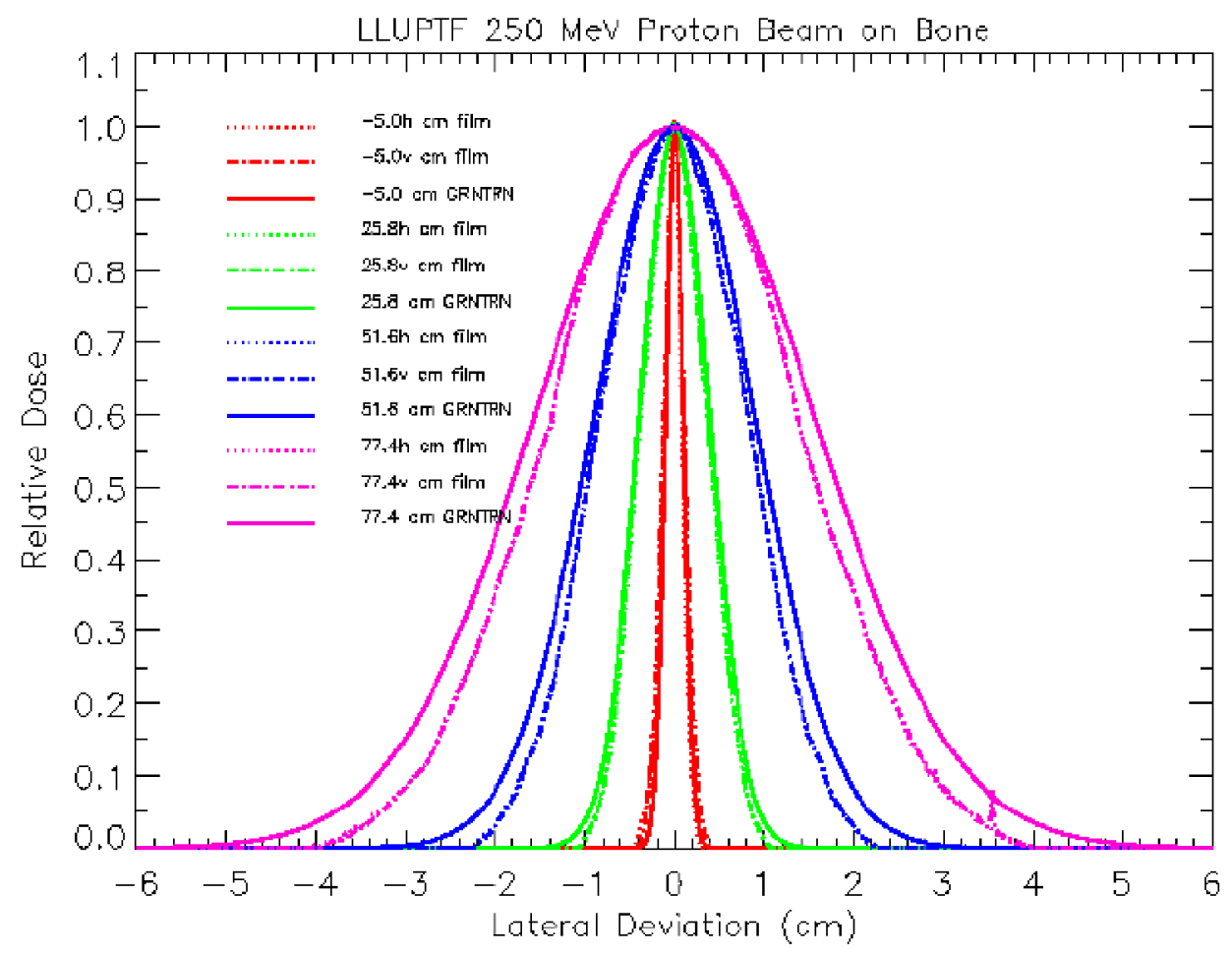

Figure 6: Lateral proton broadening in RMI bone sandwich configuration (see Figure 3). The colored lines represent results at various distances along the beamline (see Figures 1-2 for beamline distance definitions). The solid lines are GRNTRN simulations of the relative dose at lateral distances with respect to the beamline direction. The dotted and dashed lines are relative dose measured with the radiographic film in the two perpendicular (lateral) directions with respect to the beamline direction. 


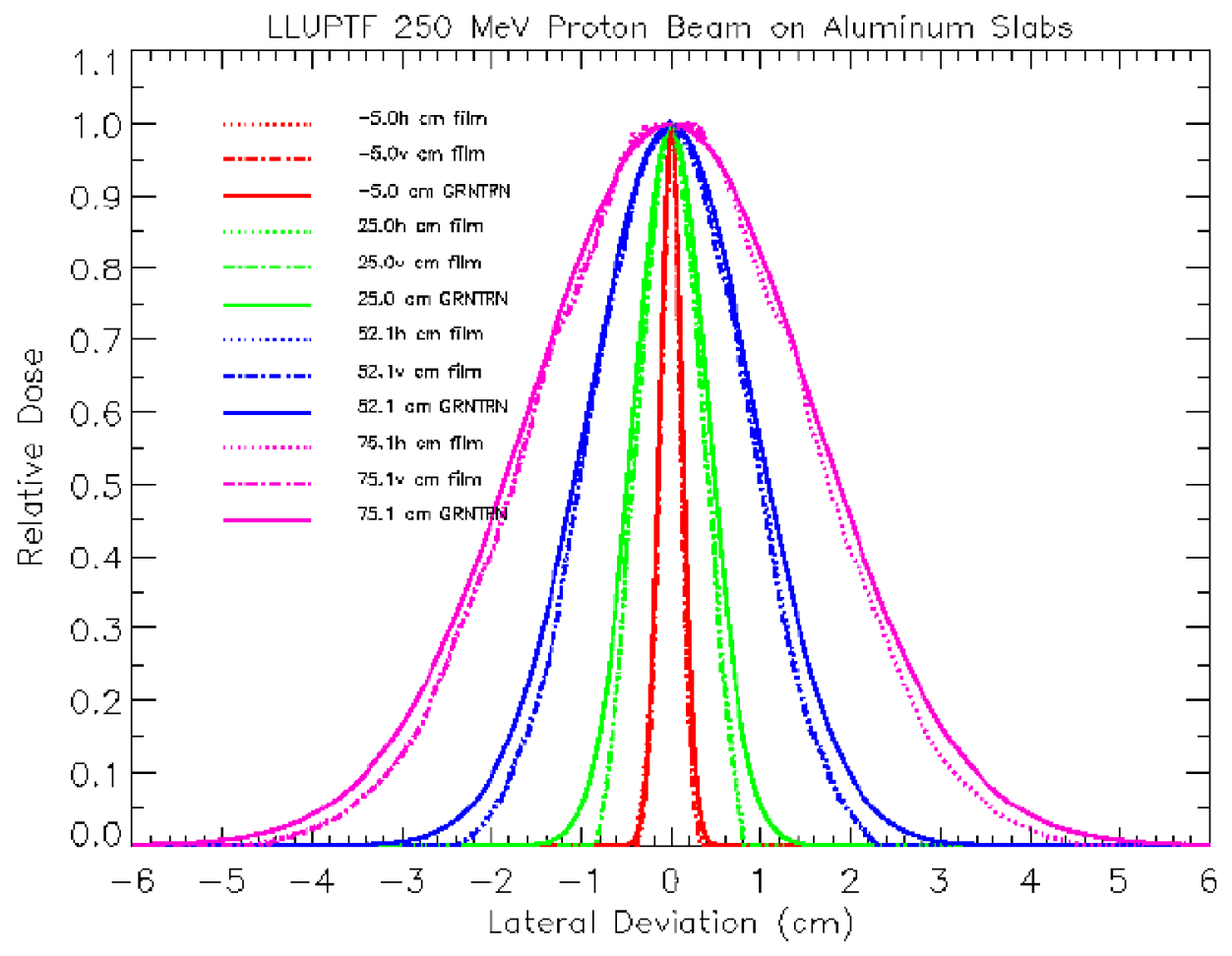

Figure 7: Lateral proton broadening in Al sandwich configuration (see Figure 3). The colored lines represent results at various distances along the beamline (see Figures 1-2 for beamline distance definitions). The solid lines are GRNTRN simulations of the relative dose at lateral distances with respect to the beamline direction. The dotted and dashed lines are relative dose measured with the radiographic film in the two perpendicular (lateral) directions with respect to the beamline direction. 


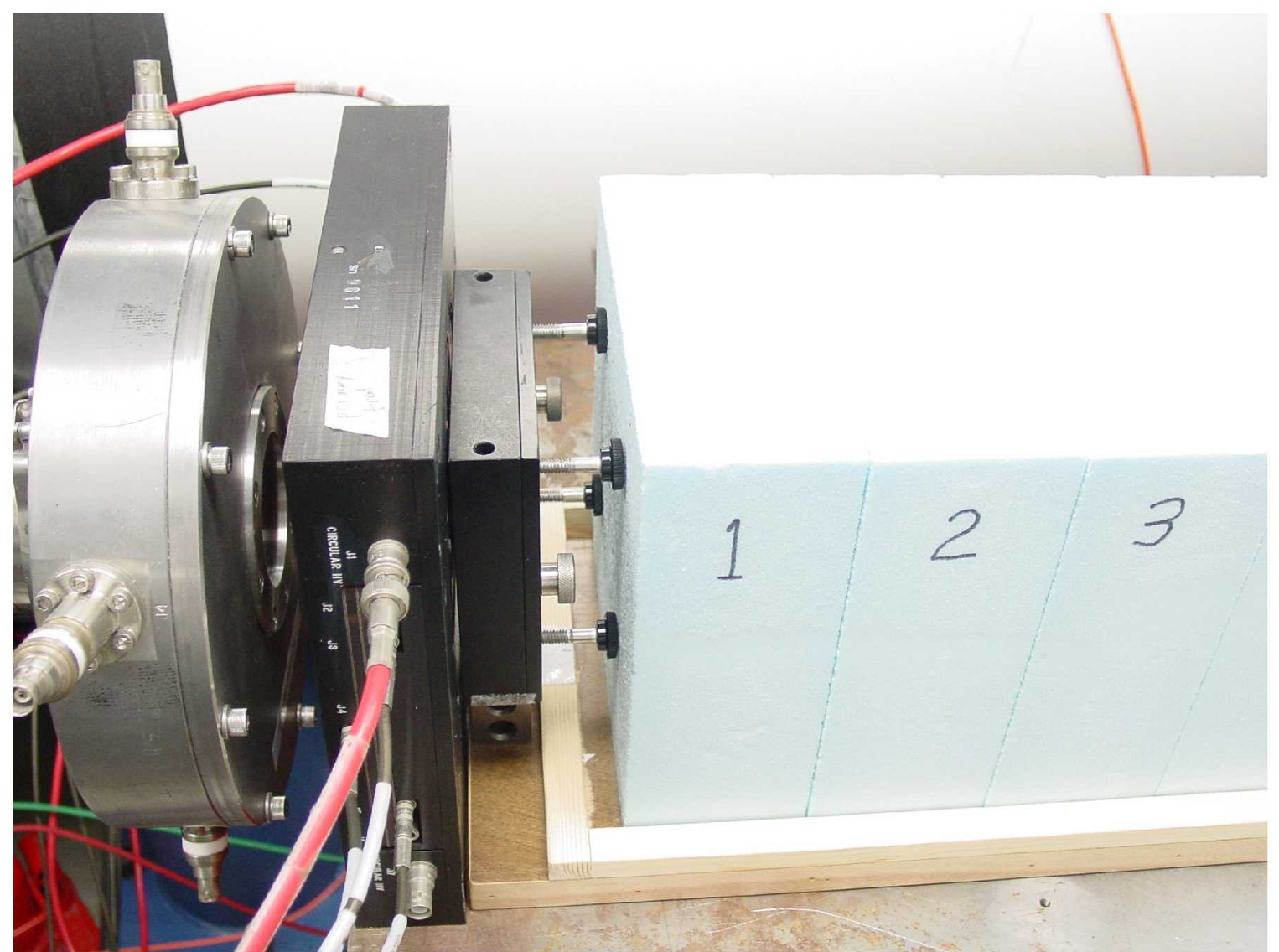

Figure 8: LLUPTF experimental set-up for proton broadening measurements in single slab configuration for the high-density (Fe and Pb) materials. In this picture, thin slabs of Fe are stacked in a single layer configuration and mounted immediately downstream of the TIC. A drift is provided by nine foam slabs downstream of the TIC. Only three foam slabs can be seen in the above picture. 


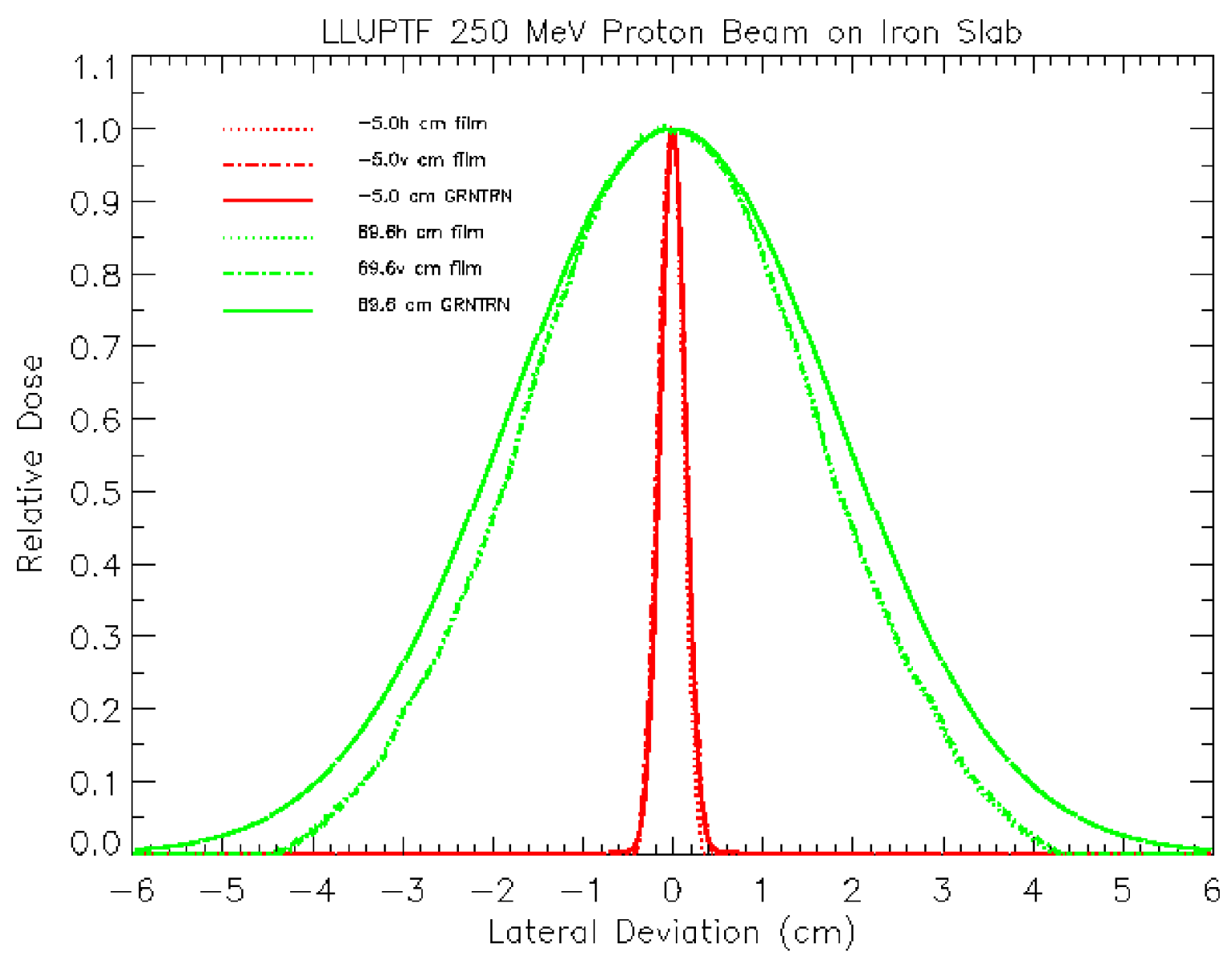

Figure 9: Lateral proton broadening of Fe single slab configuration (see Figure 8). The colored lines represent results at two distances along the beamline (see Figures 1-2 for beamline distance definitions). The solid lines are GRNTRN simulations of the relative dose at lateral distances with respect to the beamline direction. The dotted and dashed lines are relative dose measured with the radiographic film in the two perpendicular (lateral) directions with respect to the beamline direction. 


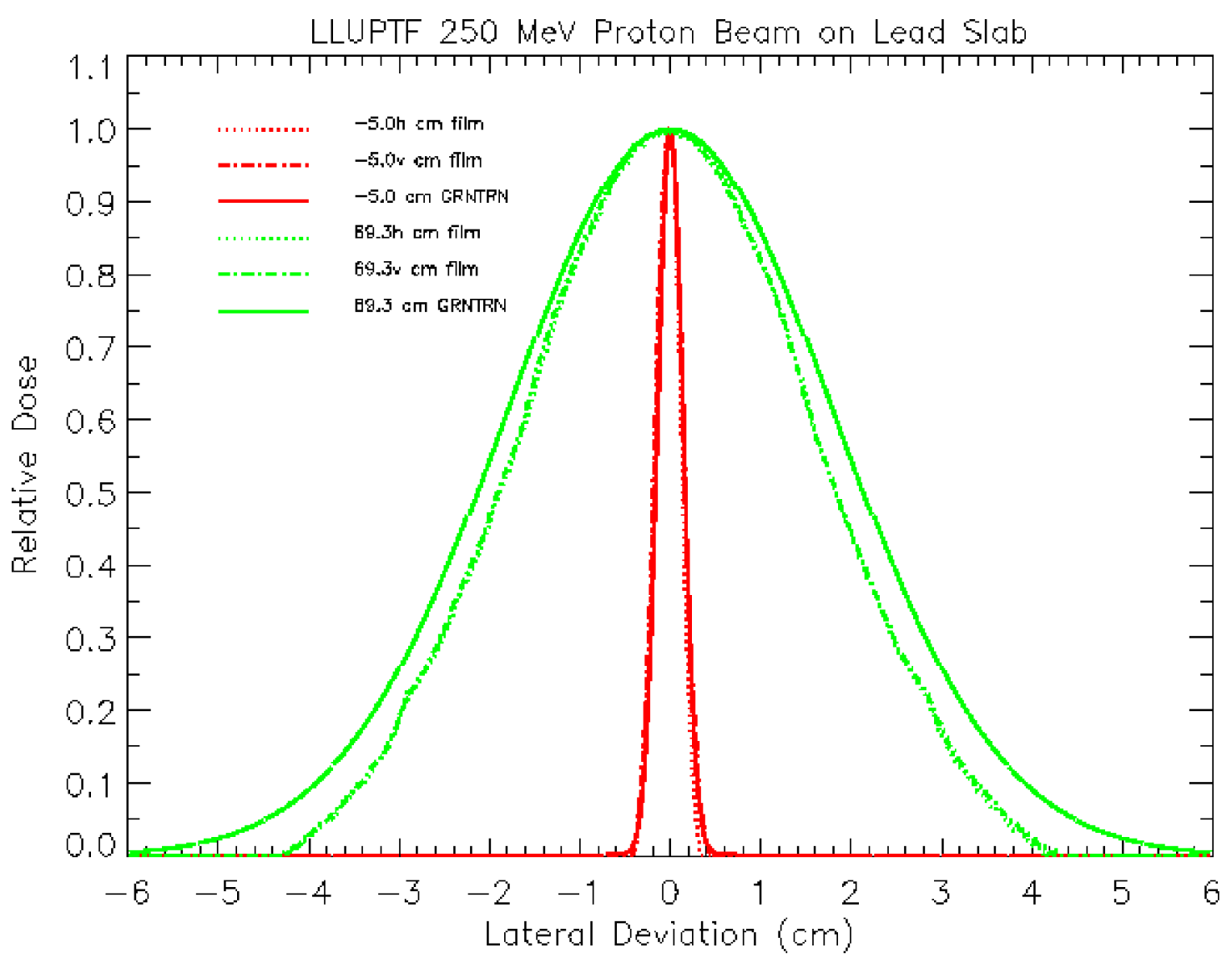

Figure 10: Lateral proton broadening of $\mathrm{Pb}$ single slab configuration (see Figure 8). The colored lines represent results at two distances along the beamline (see Figures 1-2 for beamline distance definitions). The solid lines are GRNTRN simulations of the relative dose at lateral distances with respect to the beamline direction. The dotted and dashed lines are relative dose measured with the radiographic film in the two perpendicular (lateral) directions with respect to the beamline direction. 